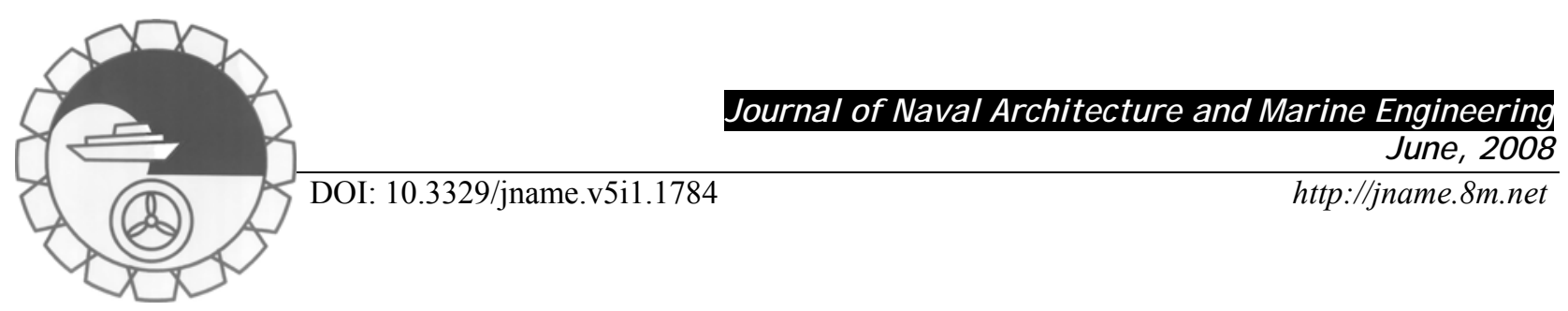

\title{
HYDROMAGNETIC THREE DIMENSIONAL COUETTE FLOW AND HEAT TRANSFER
}

\author{
S. S. Das ${ }^{1}$, M. Mohanty ${ }^{2}$, J. P. Panda ${ }^{3}$ and S. K. Sahoo ${ }^{4}$ \\ ${ }^{1}$ Department of Physics, KBDAV College, Nirakarpur, Khurda-752 019 (Orissa), India, Email: drssd2@yahoo.com \\ ${ }^{2}$ Department of Physics, Christ College, Mission Road, Cuttack-753 001 (Orissa), India \\ ${ }^{3}$ Department of Mathematics, Synergy Institute of Engg. \& Tech., Dhenkanal-759 001 (Orissa), India \\ ${ }^{4}$ Department of Computer Science \& Engg., KIIT University, Bhubaneswar-751 024 (Orissa), India
}

\begin{abstract}
This paper theoretically analyzes three dimensional couette flow of a viscous incompressible electrically conducting fluid between two infinite horizontal parallel porous flat plates in presence of a transverse magnetic field. The stationary plate and the plate in uniform motion are, respectively, subjected to a transverse sinusoidal injection and uniform suction of the fluid. The governing equations of the flow field are solved by using series expansion method and the expressions for the velocity field, the temperature field, skin friction and heat flux in terms of Nusselt number are obtained. The effects of the flow parameters on the velocity, temperature, skin friction and heat flux have been studied and analyzed with the help of figures and tables. It is observed that the magnetic parameter (M) has a retarding effect on the main velocity $(u)$ and an accelerating effect on the cross velocity $\left(w_{l}\right)$ of the flow field. The suction parameter $\left(R_{e}\right)$ has a retarding effect on the main velocity as well as on the temperature field. The Prandtl number $\left(P_{r}\right)$ reduces the temperature of the flow field and increases the rate of heat transfer at the wall $\left(N_{w}\right)$. The effect of suction parameter is to reduce the $x$-component of skin friction and to enhance the magnitude of $z$ component of the skin friction at the wall. The problem is very much significant in view of its several engineering, geophysical and industrial applications.
\end{abstract}

Keywords: Hydromagnetic, couette flow, heat transfer, three dimensions

\section{NOMENCLATURE}

$\mathrm{B}_{0} \quad$ uniform magnetic field

$l \quad$ distance between the plates

$M \quad$ magnetic parameter

$N_{u} \quad$ Nusselt number

$P_{r} \quad$ Prandtl number

$p^{*} \quad$ pressure

$R_{e} \quad$ Reynold's Number

$T^{*} \quad$ temperature

$T$ dimensionless temperature

$T_{0} \quad$ temperature at the lower plate

$T_{w} \quad$ temperature at the upper plate

$U \quad$ uniform velocity of the upper plate

$u, v, w$ dimensionless velocity components

$$
\begin{aligned}
& u^{*}, v^{*}, w^{*} \text { velocity components along } x^{*}, y^{*}, z^{*} \\
& \text { direction respectively } \\
& V \quad \text { constant suction velocity } \\
& v^{*}\left(z^{*}\right) \quad \text { sinusoidal injection velocity } \\
& x, y, z \quad \text { dimensionless Cartesian coordinates } \\
& x^{*}, y^{*}, z^{*} \quad \text { Cartesian coordinates }
\end{aligned}
$$

$\begin{array}{ll}\text { Greek Symbol } \\ \alpha & \text { thermal diffusivity } \\ \varepsilon & \text { a small positive constant }(0<\varepsilon<<1) \\ \rho & \text { density } \\ \nu & \text { kinematic viscosity } \\ \sigma & \text { electrical conductivity }\end{array}$

\section{Introduction}

The problem of hydromagnetic couette flow with heat transfer has been a subject of interest of many researchers because of its possible applications in many branches of science and technology. Channel flows have several engineering and geophysical applications, such as, in the field of chemical engineering for filtration and purification processes; in the field of agricultural engineering to study the underground water resources; in petroleum industry to study the movement of natural gas, oil and water through the oil channels and reservoirs. 
In view of these applications a series of investigations have been made by different scholars where the medium is either bounded by horizontal or vertical surfaces. Gersten and Gross (1974) studied the flow and heat transfer along a plane wall with periodic suction. Gulab and Mishra (1977) analyzed the unsteady MHD flow of a conducting fluid through a porous medium. Kaviany (1985) explained the laminar flow through a porous channel bounded by isothermal parallel plates. Vajravelu and Hadjinicolaou (1993) have investigated the heat transfer in a viscous fluid over a stretching sheet with viscous dissipation and internal heat generation. Attia and Kotb (1996) explained the MHD flow between two parallel plates with heat transfer.

The unsteady hydromagnetic natural convection in a fluid saturated porous channel was studied by Chamkha (1996). Attia (1997) analyzed the transient MHD flow and heat transfer between two parallel plates with temperature dependent viscosity. Krishna et al. (2004) presented the hydromagnetic oscillatory flow of a second order RivlinErickson fluid in a channel. Sharma and Yadav (2005) analyzed the heat transfer through three dimensional couette flow between a stationary porous plate bounded by porous medium and a moving porous plate. Sharma et al. (2005) explained the steady laminar flow and heat transfer of a non-Newtonian fluid through a straight horizontal porous channel in the presence of heat source. Recently, Jain et al. (2006) discussed the three dimensional couette flow with transpiration cooling through a porous medium in the slip flow regime.

The proposed study considers the three dimensional couette flow of a viscous incompressible electrically conducting fluid between two infinite horizontal parallel porous flat plates in presence of a transverse magnetic field. The stationary plate and the plate in uniform motion are, respectively, subjected to a transverse sinusoidal injection and uniform suction of the fluid. The governing equations of the flow field are solved by using series expansion method and the expressions for the velocity field, the temperature field, skin friction and heat flux in terms of Nusselt number are obtained. The effect of the flow parameters on the velocity field, temperature field, skin friction and Nusselt number have been studied and analyzed with the help of figures and tables.

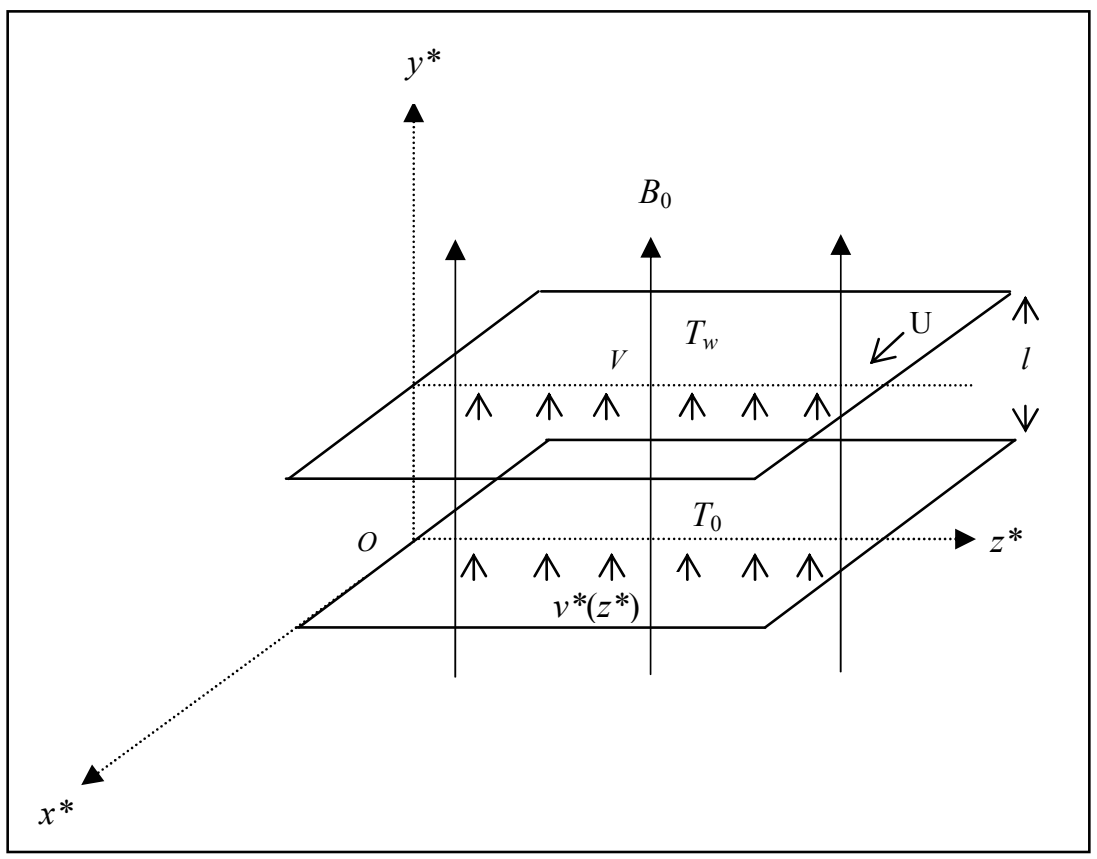

Fig. A: Physical sketch and geometry of the problem

\section{Formulation of the Problem}

Consider the three dimensional couette flow of a viscous incompressible electrically conducting fluid bounded between two infinite horizontal parallel porous plates in presence of a uniform transverse magnetic field $B_{0}$. The physical model and geometry of the problem is shown in Fig. A. A coordinate system is chosen with its origin at the 
lower stationary plate lying horizontally in $x^{*}-z^{*}$ plane and the upper plate at a distance $l$ from it is subjected to a uniform velocity $U$.

The $y^{*}$-axis is taken normal to the planes of the plates. The lower and the upper plates are assumed to be at constant temperatures $T_{0}$ and $T_{w}$, respectively, with $T_{w}>T_{0}$. The upper plate is subjected to a constant suction velocity $V$ whereas the lower plate to a transverse sinusoidal injection velocity of the form:

$$
v^{*}\left(z^{*}\right)=V\left(1+\varepsilon \cos \pi z^{*} / l\right),
$$

where $\varepsilon(<<1)$ is a very small positive constant quantity, $l$ is taken equal to the wavelength of the injection velocity.

Due to this kind of injection velocity the flow remains three dimensional. All the physical quantities involved are independent of $x^{*}$ for this fully developed laminar flow. Denoting the velocity components $u^{*}, v^{*}, w^{*}$ in $x^{*}, y^{*}, z^{*}$ directions, respectively and the temperature by $T^{*}$, the problem is governed by the following equations:

$\frac{\partial v^{*}}{\partial y^{*}}+\frac{\partial w^{*}}{\partial z^{*}}=0$

$v^{*} \frac{\partial u^{*}}{\partial y^{*}}+w^{*} \frac{\partial u^{*}}{\partial z^{*}}=v\left(\frac{\partial^{2} u^{*}}{\partial y^{* 2}}+\frac{\partial^{2} u^{*}}{\partial z^{* 2}}\right)-\frac{\sigma B_{0}^{2}}{\rho} u^{*}$,

$v^{*} \frac{\partial v^{*}}{\partial y^{*}}+w^{*} \frac{\partial v^{*}}{\partial z^{*}}=-\frac{1}{\rho} \frac{\partial p^{*}}{\partial y^{*}}+v\left(\frac{\partial^{2} v^{*}}{\partial y^{* 2}}+\frac{\partial^{2} v^{*}}{\partial z^{* 2}}\right)$,

$v^{*} \frac{\partial w^{*}}{\partial y^{*}}+w^{*} \frac{\partial w^{*}}{\partial z^{*}}=-\frac{1}{\rho} \frac{\partial p^{*}}{\partial z^{*}}+v\left(\frac{\partial^{2} w^{*}}{\partial y^{* 2}}+\frac{\partial^{2} w^{*}}{\partial z^{* 2}}\right)-\frac{\sigma B_{0}^{2}}{\rho} w^{*}$,

$v^{*} \frac{\partial T^{*}}{\partial y^{*}}+w^{*} \frac{\partial T^{*}}{\partial z^{*}}=\alpha\left(\frac{\partial^{2} T^{*}}{\partial y^{* 2}}+\frac{\partial^{2} T^{*}}{\partial z^{* 2}}\right)$,

where $\rho$ is the density, $\sigma$ is the electrical conductivity, $p^{*}$ is the pressure, $v$ is coefficient of the kinematic viscosity and $\alpha$ is the thermal diffusivity.

The boundary conditions of the problem are

$$
\begin{aligned}
& u^{*}=0, v^{*}=V\left(1+\varepsilon \cos \pi z^{*} l l\right), w^{*}=0, T^{*}=T_{0}^{*} \text { at } y^{*}=0, \\
& u^{*}=U, \quad v^{*}=V, \quad w^{*}=0, T^{*}=T_{w}^{*} \text { at } y^{*}=l .
\end{aligned}
$$

Introducing the following non-dimensional quantities,

$y=\frac{y^{*}}{l}, z=\frac{z^{*}}{l}, u=\frac{u^{*}}{U}, v=\frac{v^{*}}{V}, w=\frac{w^{*}}{V}, p=\frac{p^{*}}{\rho V^{2}}, \quad T=\frac{T^{*}-T_{0}^{*}}{T_{w}^{*}-T_{0}^{*}}$,

Equations (2) - (6) reduce to the following forms

$$
\frac{\partial v}{\partial y}+\frac{\partial w}{\partial z}=0
$$

$v \frac{\partial u}{\partial y}+w \frac{\partial u}{\partial z}=\frac{1}{R_{e}}\left(\frac{\partial^{2} u}{\partial y^{2}}+\frac{\partial^{2} u}{\partial z^{2}}\right)-\frac{M^{2}}{R_{e}} u$,

$v \frac{\partial v}{\partial y}+w \frac{\partial v}{\partial z}=-\frac{\partial p}{\partial y}+\frac{1}{R_{e}}\left(\frac{\partial^{2} v}{\partial y^{2}}+\frac{\partial^{2} v}{\partial z^{2}}\right)$,

$v \frac{\partial w}{\partial y}+w \frac{\partial w}{\partial z}=-\frac{\partial p}{\partial z}+\frac{1}{R_{e}}\left(\frac{\partial^{2} w}{\partial y^{2}}+\frac{\partial^{2} w}{\partial z^{2}}\right)-\frac{M^{2}}{R_{e}} w$, 
$v \frac{\partial T}{\partial y}+w \frac{\partial T}{\partial z}=\frac{1}{R_{e} P_{r}}\left(\frac{\partial^{2} T}{\partial y^{2}}+\frac{\partial^{2} T}{\partial z^{2}}\right)$

where $R_{e}=\frac{V l}{v}$, Reynolds number,

$$
\begin{aligned}
& M^{2}=\frac{\sigma B_{0}^{2}}{\rho} \frac{l^{2}}{v}, \text { Magnetic parameter, } \\
& P_{r}=\frac{v}{\alpha}, \text { Prandtl number. }
\end{aligned}
$$

The corresponding boundary conditions become

$u=0, \quad v=1+\varepsilon \cos \pi z, \quad w=0, \quad T=0$ at $y=0$,

$u=1, \quad v=1, \quad \quad w=0, \quad T=1$ at $y=1$.

\section{Method of Solution}

In order to solve the problem, we assume the solutions of the following form because the amplitude $\quad 0<\varepsilon<<1$ of the permeability variation is very small:

$$
\begin{aligned}
& u(y, z)=u_{0}(y)+\varepsilon u_{1}(y, z)+\varepsilon^{2} u_{2}(y, z)+\ldots \ldots \\
& v(y, z)=v_{0}(y)+\varepsilon v_{1}(y, z)+\varepsilon^{2} v_{2}(y, z)+\ldots \ldots \\
& w(y, z)=w_{0}(y)+\varepsilon w_{1}(y, z)+\varepsilon^{2} w_{2}(y, z)+\ldots \ldots \\
& p(y, z)=p_{0}(y)+\varepsilon p_{1}(y, z)+\varepsilon^{2} p_{2}(y, z)+\ldots \ldots \\
& T(y, z)=T_{0}(y)+\varepsilon T_{1}(y, z)+\varepsilon^{2} T_{2}(y, z)+\ldots \ldots
\end{aligned}
$$

When $\varepsilon=0$, the problem reduces to the two dimensional free convective MHD flow which is governed by the following equations:

$$
\begin{aligned}
& \frac{d v_{0}}{d y}=0, \\
& \frac{d^{2} u_{0}}{d y^{2}}-v_{0} R_{e} \frac{d u_{0}}{d y}-M^{2} u_{0}=0, \\
& \frac{d^{2} T_{0}}{d y^{2}}-v_{0} R_{e} P_{r} \frac{d T_{0}}{d y}=0 .
\end{aligned}
$$

The corresponding boundary conditions become

$u_{0}=0, \quad v_{0}=1, \quad T_{0}=0$ at $y=0$,

$u_{0}=1, \quad v_{0}=1, \quad T_{0}=1$ at $y=1$.

The solutions of these equations for this two dimensional problem are

$$
\begin{aligned}
& u_{0}(y)=\frac{e^{\lambda_{1} y}-e^{\lambda_{2} y}}{e^{\lambda_{1}}-e^{\lambda_{2}}}, \\
& T_{0}(y)=\frac{e^{R_{e} P_{r} y}-1}{e^{R_{e} P_{r}}-1},
\end{aligned}
$$

with $\quad v_{0}=1, w_{0}=0, p_{0}=$ constant,

$$
\lambda_{1}=\frac{1}{2}\left[R_{e}+\sqrt{R_{e}^{2}+4 M^{2}}\right], \quad \lambda_{2}=\frac{1}{2}\left[R_{e}-\sqrt{R_{e}^{2}+4 M^{2}}\right] .
$$

When $\varepsilon \neq 0$, substituting (16) into Equations (9) - (13) and comparing the coefficients of like powers of $\varepsilon$, neglecting those of $\varepsilon^{2}$, we get the following first order equations with the help of Equation (23): 


$$
\begin{aligned}
& \frac{\partial v_{1}}{\partial y}+\frac{\partial w_{1}}{\partial z}=0, \\
& v_{1} \frac{\partial u_{0}}{\partial y}+\frac{\partial u_{1}}{\partial y}=\frac{1}{R_{e}}\left(\frac{\partial^{2} u_{1}}{\partial y^{2}}+\frac{\partial^{2} u_{1}}{\partial z^{2}}\right)-\frac{1}{R_{e}} M^{2} u_{1}, \\
& \frac{\partial v_{1}}{\partial y}=-\frac{\partial p_{1}}{\partial y}+\frac{1}{R_{e}}\left(\frac{\partial^{2} v_{1}}{\partial y^{2}}+\frac{\partial^{2} v_{1}}{\partial z^{2}}\right), \\
& \frac{\partial w_{1}}{\partial y}=-\frac{\partial p_{1}}{\partial z}+\frac{1}{R_{e}}\left(\frac{\partial^{2} w_{1}}{\partial y^{2}}+\frac{\partial^{2} w_{1}}{\partial z^{2}}\right)-\frac{1}{R_{e}} M^{2} w_{1}, \\
& v_{1} \frac{\partial T_{0}}{\partial y}+\frac{\partial T_{1}}{\partial y}=\frac{1}{R_{e} P_{r}}\left(\frac{\partial^{2} T_{1}}{\partial y^{2}}+\frac{\partial^{2} T_{1}}{\partial z^{2}}\right),
\end{aligned}
$$

The corresponding boundary conditions are

$u_{1}=0, \quad v_{1}=\cos \pi z, \quad w_{1}=0, \quad T_{1}=0$ at $y=0$,

$u_{1}=0, \quad v_{1}=0, \quad w_{1}=0, \quad T_{1}=0$ at $y=1$.

Equations (24)-(28) are the linear partial differential equations which describe the MHD three-dimensional flow through a porous medium. For solution we shall first consider three Equations (24), (26) and (27) being independent of the main flow component $u_{1}$ and the temperature field $T_{1}$. We assume $v_{1}, w_{1}$ and $p_{1}$ of the following form:

$v_{1}(y, z)=v_{11}(y) \cos \pi z$

$w_{1}(y, z)=\frac{1}{\pi} v_{11}^{\prime}(y) \sin \pi z$,

$p_{1}(y, z)=p_{11}(y) \cos \pi z$

where the prime in $v_{11}^{\prime}(y)$ denotes the differentiation with respect to $\mathrm{y}$. Expressions for $v_{1}(y, z)$ and $w_{1}(y, z)$ have been chosen so that the equation of continuity (24) is satisfied.

Substituting these expressions (30)-(32) into (26) and (27) and solving under corresponding transformed boundary conditions, we get the solutions of $v_{1}, w_{1}$ and $p_{1}$ as:

$$
\begin{aligned}
& v_{1}(y, z)=\frac{1}{A}\left[A_{1} e^{m_{1} y}+A_{2} e^{m_{2} y}+A_{3} e^{\pi y}+A_{4} e^{-\pi y}\right] \cos \pi z, \\
& w_{1}(y, z)=-\frac{1}{\pi A}\left[A_{1} m_{1} e^{m_{1} y}+A_{2} m_{2} e^{m_{2} y}+A_{3} \pi e^{\pi y}-A_{4} \pi e^{-\pi y}\right] \sin \pi z, \\
& p_{1}(y, z)=-\frac{1}{\pi R_{e} A}\left[A_{3}\left(\pi R_{e}+M^{2}\right) e^{\pi y}+A_{4}\left(\pi R_{e}-M^{2}\right) e^{-\pi y}\right] \cos \pi z,
\end{aligned}
$$

where

$$
\begin{aligned}
& m_{1}=\frac{R_{e}}{2}+\sqrt{\frac{R_{e}^{2}}{4}+\left(\pi^{2}+M^{2}\right)}, \quad m_{2}=\frac{R_{e}}{2}-\sqrt{\frac{R_{e}^{2}}{4}+\left(\pi^{2}+M^{2}\right)}, \\
& \left.\left.A=\left(\pi-m_{1}\right)\left(\pi+m_{2}\right) \mid e^{m_{2}-\pi}+e^{m_{1}+\pi}\right\rfloor+\left(m_{1}+\pi\right)\left(m_{2}-\pi\right)\left[e^{\pi+m_{2}}+e^{m_{1}-\pi}\right]-2 \pi\left(m_{2}-m_{1}\right) \mid e^{m_{1}+m_{2}}+1\right], \\
& A_{1}=-2 \pi m_{2}+\pi\left(m_{2}+\pi\right) e^{\pi+m_{2}}-\pi\left(\pi-m_{2}\right) e^{m_{2}-\pi}, \\
& A_{2}=2 \pi m_{1}-\pi\left(\pi+m_{1}\right) e^{m_{1}-\pi}-\pi\left(m_{1}-\pi\right) e^{m_{1}+\pi},
\end{aligned}
$$




$$
\begin{aligned}
& A_{3}=-m_{1}\left(m_{2}+\pi\right) e^{m_{2}-\pi}+m_{2}\left(m_{1}+\pi\right) e^{m_{1}-\pi}-\pi\left(m_{2}-m_{1}\right) e^{m_{1}+m_{2}}, \\
& A_{4}=m_{1}\left(m_{2}-\pi\right) e^{m_{2}+\pi}-m_{2}\left(m_{1}-\pi\right) e^{m_{1}+\pi}-\pi\left(m_{2}-m_{1}\right) e^{m_{1}+m_{2}} .
\end{aligned}
$$

To solve Equations (25) and (28) for $u_{1}$ and $T_{1}$, we assume

$$
u_{1}(y)=u_{11}(y) \cos \pi z
$$

$T_{1}(y, z)=T_{11}(y) \cos \pi z$.

Substituting the values of $u_{1}$ and $T_{1}$ from Equations (36) and (37) into Equations (25) and (28), we get

$u_{11}^{\prime \prime}-R_{e} u_{11}^{\prime}-\left(\pi^{2}+M^{2}\right) u_{11}=R_{e} v_{11} u_{0}$,

$T_{11}^{\prime \prime}-R_{e} P_{r} T_{11}^{\prime}-\pi^{2} T_{11}=R_{e} P_{r} v_{11} T_{0}$,

where the primes denote the differentiation with respect to $y$.

The corresponding boundary conditions are

$y=0: u_{11}=0, \quad T_{11}=0$,

$y=1: u_{11}=0, \quad T_{11}=0$.

Solving Equations (38) and (39) under the boundary conditions (40) and using Equations (36) and (37), we get

$$
\begin{aligned}
u_{1} & =\left[B_{1} e^{-m_{3} y}-B_{2} e^{m_{4} y}+B_{3} e^{2 m_{1} y}+B_{4} e^{\left(m_{1}+m_{2}\right) y}-B_{5} e^{2 m_{2} y}+B_{6} e^{\left(m_{1}+\pi\right) y}\right. \\
& \left.-B_{7} e^{\left(m_{1}-\pi\right) y}-B_{8} e^{\left(m_{2}+\pi\right) y}+B_{9} e^{\left(m_{2}-\pi\right) y}\right] \cos \pi z \\
T_{1}= & {\left[D_{1} e^{m_{5} y}+D_{2} e^{m_{6} y}+D_{3} e^{\left(m_{1}+R_{e} P_{r}\right) y}+D_{4} e^{\left(m_{2}+R_{e} P_{r}\right) y}+D_{5} e^{\left(m_{2}+R_{e} P_{r}\right) y}+D_{6} e^{\left(\pi+R_{e} P_{r}\right) y}\right] \cos \pi z }
\end{aligned}
$$

where

$$
\begin{aligned}
& m_{3}=-\frac{R_{e}}{2}+\sqrt{\frac{R_{e}^{2}}{4}+\left(\pi^{2}+M^{2}\right)}, m_{4}=\frac{R_{e}}{2}+\sqrt{\frac{R_{e}^{2}}{4}+\left(\pi^{2}+M^{2}\right)}, m_{5}=\frac{R_{e} P_{r}}{2}+\sqrt{\frac{R_{e}^{2} P_{r}^{2}}{4}+\pi^{2}}, \\
& m_{6}=\frac{R_{e} P_{r}}{2}-\sqrt{\frac{R_{e}^{2} P_{r}^{2}}{4}+\pi^{2}}, A_{5}=B_{3}+B_{4}-B_{5}+B_{6}-B_{7}-B_{8}+B_{9}, \\
& A_{6}=B_{3} e^{2 m_{1}}+B_{4} e^{m_{1}+m_{2}}-B_{5} e^{2 m_{2}}+\pi B_{6} e^{m_{1}+\pi}-B_{7} e^{m_{1}-\pi}+B_{6} e^{m_{1}+\pi}-B_{7} e^{m_{1}-\pi}, \\
& A_{7}=D_{3}+D_{4}+D_{6}+D_{7}, A_{8}=D_{3} e^{m_{1}+R_{e} P_{r}}+D_{4} e^{m_{2}+R_{e} P_{r}}+D_{6} e^{\pi+R_{e} P_{r}}+D_{7} e^{-\pi+R_{e} P_{r}}, \\
& B_{1}=\left(\frac{A_{5}-A_{6} e^{m_{3}}}{1-e^{m_{3}+m_{4}}}-A_{5}\right), B_{2}=\left(\frac{A_{5}-A_{6} e^{m_{3}}}{1-e^{m_{3}+m_{4}}}\right), B_{3}=\frac{R_{e} A_{1}}{A m_{1}\left(e^{\lambda_{1}}-e^{\lambda_{2}}\right)\left(2 m_{1}+m_{3}\right)} \text {, } \\
& B_{4}=\frac{R_{e}\left(A_{2}-A_{1}\right)}{A m_{2}\left(e^{\lambda_{1}}-e^{\lambda_{2}}\right)\left(m_{1}+m_{2}+m_{3}\right)}, B_{5}=\frac{R_{e} A_{2}}{A\left(e^{\lambda_{1}}-e^{\lambda_{2}}\right)\left(2 m_{2}+m_{3}\right)\left(2 m_{2}-m_{4}\right)}, \\
& B_{6}=\frac{R_{e} A_{3}}{\pi A\left(e^{\lambda_{1}}-e^{\lambda_{2}}\right)\left(m_{1}+\pi+m_{3}\right)}, B_{7}=\frac{R_{e} A_{4}}{\pi A\left(e^{\lambda_{1}}-e^{\lambda_{2}}\right)\left(m_{1}-\pi+m_{3}\right)}, \\
& B_{8}=\frac{R_{e} A_{3}}{A\left(e^{\lambda_{1}}-e^{\lambda_{2}}\right)\left(m_{2}+\pi+m_{3}\right)\left(m_{2}+\pi-m_{4}\right)}, B_{9}=\frac{R_{e} A_{4}}{A\left(e^{\lambda_{1}}-e^{\lambda_{2}}\right)\left(m_{2}-\pi+m_{3}\right)\left(m_{2}-\pi-m_{4}\right)}, \\
& D_{1}=\left(-A_{7}-\frac{A_{7}-A_{8} e^{-m_{5}}}{e^{m_{6}-m_{5}}-1}\right), D_{2}\left(\frac{A_{7}-A_{8} e^{-m_{5}}}{e^{m_{6}-m_{5}}-1}\right), D_{3}=\frac{A_{1} R_{e}^{2} P_{r}^{2}}{A\left(e^{R_{e} P_{r}}-1\right)\left(m_{1}+R_{e} P_{r}-m_{5}\right)\left(m_{1}+R_{e} P_{r}-m_{6}\right)} \text {, } \\
& D_{4}=\frac{A_{2} R_{e}^{2} P_{r}^{2}}{A\left(e^{R_{e} P_{r}}-1\right)\left(m_{2}+R_{e} P_{r}-m_{5}\right)\left(m_{2}+R_{e} P_{r}-m_{6}\right)}, D_{5}=\frac{A_{3} R_{e}^{2} P_{r}^{2}}{A\left(e^{R_{e} P_{r}}-1\right)\left(m_{2}+R_{e} P_{r}-m_{5}\right)\left(m_{2}+R_{e} P_{r}-m_{6}\right)},
\end{aligned}
$$




$$
D_{6}=\frac{A_{3} R_{e}^{2} P_{r}^{2}}{A\left(e^{R_{e} P_{r}}-1\right)\left(\pi+R_{e} P_{r}-m_{5}\right)\left(\pi+R_{e} P_{r}-m_{6}\right)}, D_{7}=\frac{A_{4} R_{e}^{2} P_{r}^{2}}{A\left(e^{R_{e} P_{r}}-1\right)\left(R_{e} P_{r}-\pi-m_{5}\right)\left(R_{e} P_{r}-\pi-m_{6}\right)} .
$$

Substituting the values of $u_{0}, u_{1}, T_{0}$ and $T_{1}$ from Equations (21), (41), (22) and (42) in Equation (16), the solutions for velocity and temperature are given by

$$
\begin{aligned}
u & =\frac{e^{\lambda_{1} y}-e^{\lambda_{2} y}}{e^{\lambda_{1}}-e^{\lambda_{2}}}+\varepsilon\left[B_{1} e^{-m_{3} y}-B_{2} e^{m_{4} y}+B_{3} e^{2 m_{1} y}+B_{4} e^{\left(m_{1}+m_{2}\right) y}-B_{5} e^{2 m_{2} y}+B_{6} e^{\left(m_{1}+\pi\right) y}\right. \\
& \left.-B_{7} e^{\left(m_{1}-\pi\right) y}-B_{8} e^{\left(m_{2}+\pi\right) y}+B_{9} e^{\left(m_{2}-\pi\right) y}\right] \cos \pi z+O\left(\varepsilon^{2}\right) \\
T & =\frac{e^{R_{e} P_{r} y}-1}{e^{R_{e} P_{r}}-1}+\varepsilon\left[D_{1} e^{m_{5} y}+D_{2} e^{m_{6} y}+D_{3} e^{\left(m_{1}+R_{e} P_{r}\right) y}\right. \\
& \left.+D_{4} e^{\left(m_{2}+R_{e} P_{r}\right) y}+D_{5} e^{\left(m_{2}+R_{e} P_{r}\right) y}+D_{6} e^{\left(\pi+R_{e} P_{r}\right) y}\right] \cos \pi z+O\left(\varepsilon^{2}\right)
\end{aligned}
$$

\section{Skin Friction}

The $x$ - and $z$-components of skin friction at the wall are given by

$$
\tau_{x}=\left(\frac{d u_{0}}{d y}\right)_{y=0}+\varepsilon\left(\frac{d u_{1}}{d y}\right)_{y=0} \text { and } \tau_{z}=\varepsilon\left(\frac{d w_{1}}{d y}\right)_{y=0} .
$$

\section{Rate of Heat Transfer}

The rate of heat transfer i.e. heat flux at the wall in terms of Nusselt number $\left(N_{\mathrm{u}}\right)$ is given by

$$
N_{u}=\left(\frac{d T_{0}}{d y}\right)_{y=0}+\varepsilon\left(\frac{d T_{1}}{d y}\right)_{y=0}
$$

\section{Results and Discussion}

The hydromagnetic three dimensional couette flow of a viscous incompressible electrically conducting fluid between two infinite horizontal parallel porous flat plates with heat transfer has been analyzed. The governing equations of the flow field are solved by using series expansion method and the expressions for the velocity field, temperature field, skin friction and heat flux in terms of Nusselt number are obtained. The effect of the flow parameters on the velocity field and temperature field have been studied and discussed with the help of velocity profiles shown in Figs. 1-3 and temperature profiles shown in Figs. 4-5 and the effects of the flow parameters on the skin friction and heat flux have been discussed with the help of Tables 1 and 2 respectively.

\subsection{Main velocity field}

The major change in the main velocity $(u)$ of the flow field is due to the variation of magnetic parameter $(M)$ and suction / injection parameter $\left(R_{\mathrm{e}}\right)$. The magnetic parameter affects the main velocity of the flow field to a greater extent than the suction / injection parameter. The effects of these parameters have been presented in Figs. 1 and 2 respectively.

\subsubsection{Effect of suction / injection parameter $\left(R_{\mathrm{e}}\right)$}

In Fig. 1, we present the variation in the main velocity of the flow field due to the change of the suction / injection parameter keeping other parameters of the flow field constant. It is observed that the suction / injection parameter retards the velocity of the flow field at all points. As the suction / injection of the fluid through the plate increases, the plate is cooled down and in consequence of which the viscosity of the flowing fluid increases. Therefore, there is a gradual decrease in velocity of the fluid as $R_{e}$ increases. Further, the velocity increases slowly from zero to its 
maximum value as we proceed from the inlet section. But in absence of suction / injection $\left(R_{\mathrm{e}}=0\right)$, there is a rapid increase in velocity and the velocity is proportional to the distance from the inlet section.

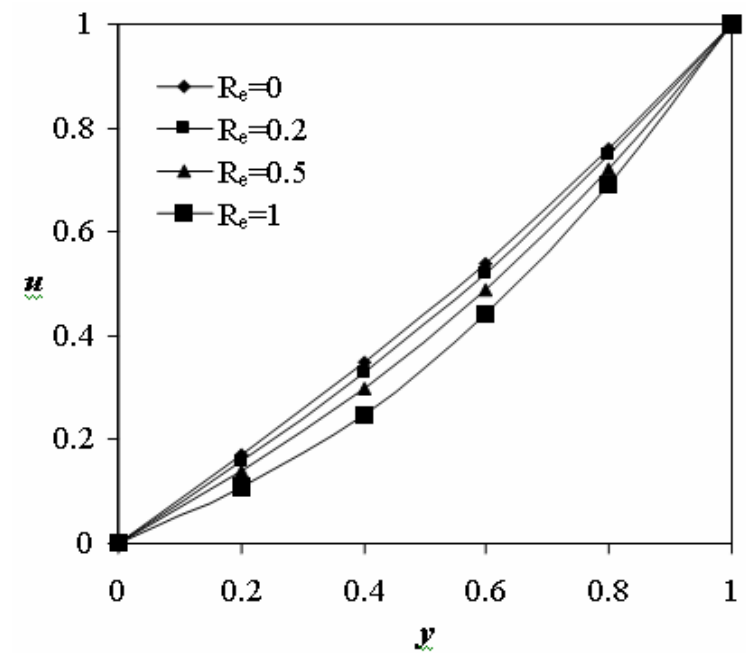

Fig. 1: Velocity Profile against y for different values of $\mathrm{R}_{\mathrm{e}}$ with $\mathrm{z}=0, \varepsilon=0.02, \mathrm{M}=1$

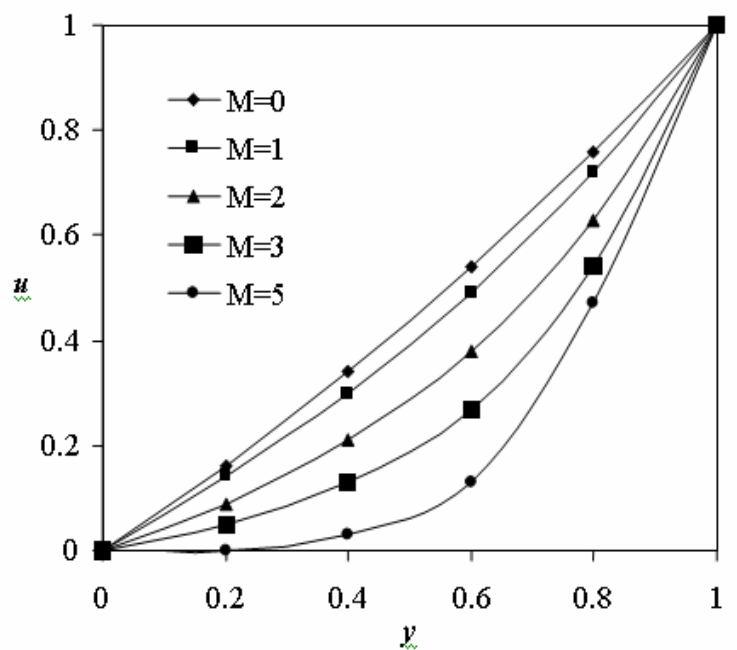

Fig. 2: Velocity Profile against y for different values of $\mathrm{M}$ with $\mathrm{z}=0, \varepsilon=0.02, \mathrm{R}_{\mathrm{e}}=0.2$

\subsubsection{Effect of magnetic parameter (M)}

Fig. 2 depicts the effect of the magnetic parameter on the main velocity of the flow field. The curve with $M=0$ corresponds to the flow in absence of magnetic field. The main velocity is observed to increase slowly from zero to its maximum value as we proceed from the inlet section. But in absence of magnetic field $(M=0)$, there is a uniform variation in the velocity of the flow field. Comparing the curves of Fig. 2, it is observed that the magnetic parameter has a retarding effect on the main velocity of the flow field due to the action of Lorentz force on the flow field. Further, comparing the curves of Figs. 1 and 2 it is observed that the magnetic parameter has a very dominant effect on the main velocity field over the suction / injection parameter.

\subsection{Cross flow velocity field}

The variation in the magnitude of the cross flow velocity $\left(w_{1}\right)$ of the flow field is shown in Fig. 3 for three different values of the magnetic parameter $(M=$ $3,5,10)$. The magnetic parameter has an accelerating effect on the cross velocity of the flow field near the lower plate. It is further observed that the cross velocity at first increases sharply to a peak value and then decreases to zero.

\subsection{Temperature field}

The temperature of the flow field is affected by the variation of Prandtl number and the suction / injection parameter. These variations are shown in Figs. 4 and 5 respectively. The suction / injection parameter affects the temperature field to a greater extent than the Prandtl number.

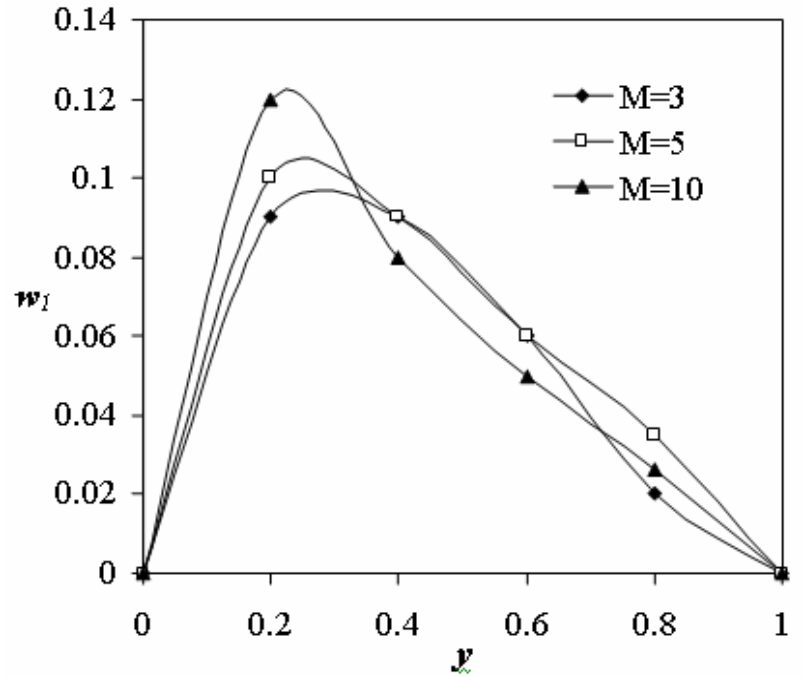

Fig. 3: Cross flow velocity profile against $y$ for different values of $\mathrm{M}$ with $\mathrm{z}=0.5, \varepsilon=0.02$, $\mathrm{R}_{\mathrm{e}}=0.2$ 


\subsubsection{Effect of Prandtl number $\left(P_{r}\right)$}

In Fig. 4, we discuss the effect of Prandtl number $\left(P_{\mathrm{r}}\right)$ on the temperature of the flow field. Fig. 4 is a plot of temperature against the non-dimensional distance for three different values of $P_{\mathrm{r}}(=0.71,1,2)$. A comparison of the curves of the said figure shows that the Prandtl number decreases the temperature at all points of the flow field. With the increase of Prandtl number, the thermal conduction in the flow field is lowered and the viscosity of the flowing fluid becomes higher. Consequently, the molecular motion of the fluid elements is lowered down and therefore, the flow field suffers a decrease in temperature at all points as we increase $\mathrm{P}_{\mathrm{r}}$.

\subsubsection{Effect of suction / injection parameter $\left(R_{\mathrm{e}}\right)$}

The effect of suction / injection parameter on the temperature of the flow field is shown in Fig. 5. The temperature of the flow field is found to decrease in presence of growing suction / injection. The temperature profile becomes very much linear in absence of suction / injection $\left(R_{\mathrm{e}}=0\right)$. In presence of higher suction / injection more amount of fluid is pushed into the flow field through the plate due to which the flow field suffers a decrease in temperature of the flow field at all points.

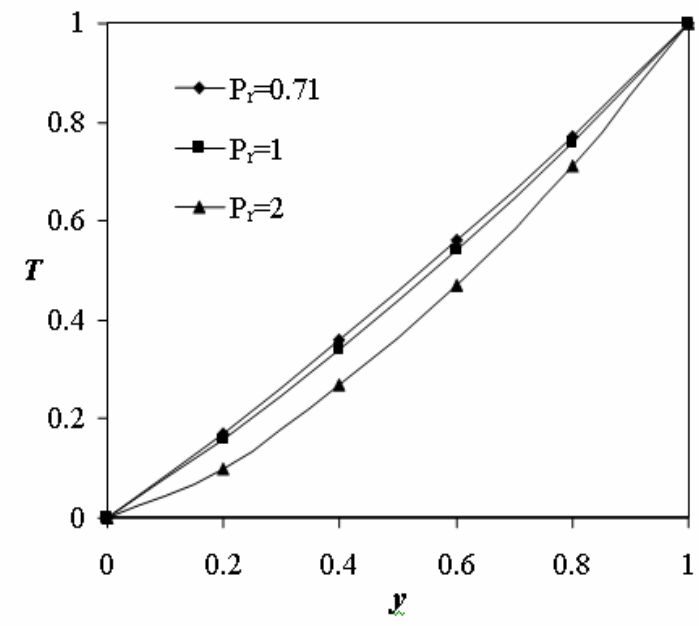

Fig. 4: Temperature profile against $\mathrm{y}$ for different values of $\mathrm{P}_{\mathrm{r}}$ with $\mathrm{z}=0, \varepsilon=0.02, \mathrm{M}=1, \mathrm{R}_{\mathrm{e}}=0.5$

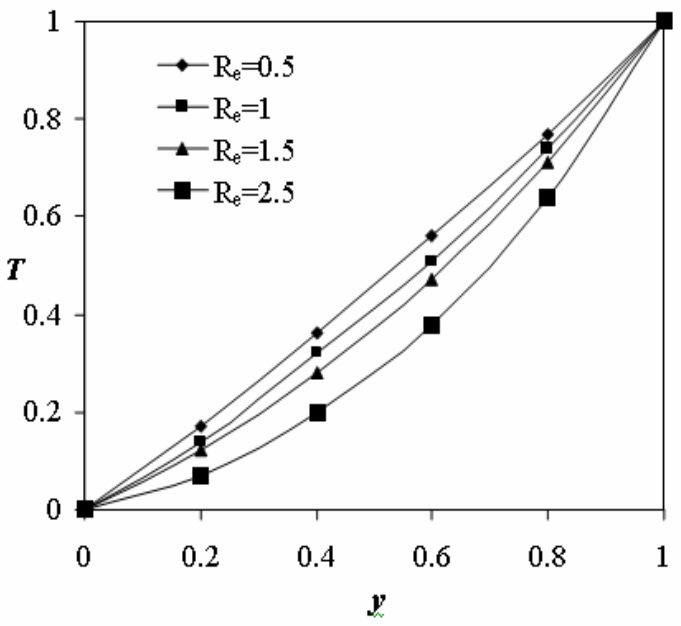

Fig. 5: Temperature profile against $y$ for different values of $\mathrm{R}_{\mathrm{e}}$ with $\mathrm{z}=0, \varepsilon=0.02, \mathrm{M}=1, \mathrm{P}_{\mathrm{r}}=0.71$

\subsection{Skin friction}

The skin friction at the wall for different values of suction / injection parameter $\left(R_{\mathrm{e}}\right)$ has been entered in Table 1 . The suction / injection parameter reduces the skin friction at the wall in $\mathrm{x}$-direction while it enhances the magnitude of $z$-component of the skin friction at the wall.

Table 1: Values of skin friction at the wall for different values of suction / injection parameter $\left(R_{\mathrm{e}}\right)$

\begin{tabular}{|c|c|c|}
\hline \multicolumn{1}{|l|}{$R_{\mathrm{e}}$} & \multicolumn{1}{|c|}{$\tau_{\mathrm{x}}$} & \multicolumn{1}{c|}{$\tau_{\mathrm{z}}$} \\
\hline 0 & 0.8509 & -0.0773 \\
\hline 0.01 & 0.8467 & -0.0817 \\
\hline 0.2 & 0.7616 & -0.7228 \\
\hline 0.5 & 0.6785 & 1.3491 \\
\hline
\end{tabular}

Table 2: Values of rate of heat transfer at the wall for different values of Prandtl number $\left(\mathrm{P}_{\mathrm{r}}\right)$

\begin{tabular}{|c|c|}
\hline$P_{\mathrm{r}}$ & $N_{\mathrm{u}}$ \\
\hline 0.71 & -1.5030 \\
\hline 1 & -0.7494 \\
\hline 2 & 0.0936 \\
\hline 7 & 0.1437 \\
\hline
\end{tabular}

\subsection{Rate of heat transfer}

The rate of heat transfer in terms of Nusselt number $\left(N_{\mathrm{u}}\right)$ for different values of the Prandtl number $\left(P_{\mathrm{r}}\right)$ is presented in Table 2. The Prandtl number $\left(P_{\mathrm{r}}\right)$ is found to enhance the rate of heat transfer at the wall. It is interesting to 
observe that for lower value of $P_{\mathrm{r}}(\leq 1)$, the rate of heat transfer assumes negative values while for higher values $\left(P_{\mathrm{r}}\right.$ $\geq 1$ ), it takes positive values.

\section{Conclusion}

The present analysis brings out the following interesting results of physical interest on the velocity and temperature of the flow field:

i) The magnetic parameter $(M)$ retards the main velocity $(u)$ at all points of the flow field due to the magnetic pull of the Lorentz force acting on the flow field and accelerates the cross velocity $\left(w_{1}\right)$ of the flow field.

ii) The suction / injection parameter $\left(R_{\mathrm{e}}\right)$ decelerates the main velocity of the flow field while no appreciable effect is observed for cross velocity of the flow field.

iii) The Prandtl number $\left(P_{\mathrm{r}}\right)$ reduces the temperature of the flow field at all points.

iv) The suction / injection parameter $\left(R_{\mathrm{e}}\right)$ diminishes the temperature of the flow field at all points.

v) The suction / injection parameter reduces the $x$-component of skin friction and enhances the magnitude of $z$ component of the skin friction at the wall.

vi) The rate of heat transfer at the wall $\left(N_{\mathrm{u}}\right)$ increases with the increase of the Prandtl number $\left(P_{\mathrm{r}}\right)$ of the flow field.

\section{References}

Attia, H. A. (1997): Transient MHD Flow and Heat Transfer between Two Parallel Plates with Temperature Dependent Viscosity, Mech. Res. Commun. 26, 115-121. doi:10.1016/S0093-6413(98)00108-6

Attia, H. A. and Kotb, N. A. (1996): MHD Flow Between Two Parallel Plates With Heat Transfer, Acta Mech. 117, 215-220. doi:10.1007/BF01181049

Chamkha, A. J. (1996): Unsteady Hydromagnetic Natural Convection in a Fluid Saturated Porous Medium Channel, Advances Filtra. Sep. Tech. 10, 369-375.

Gersten, K. and Gross, J. F. (1974): Flow and Heat Transfer Along a Plane Wall With Periodic Suction, Z. Angew. Math. Phys. 25 (3), 399-408. doi:10.1007/BF01594956

Gulab, R. and Mishra, R. S. (1977): Unsteady Flow Through Magnetohydrodynamic Porous Media, Ind. J. Pure Appl. Math. 8, 637-642.

Kaviany, M. (1985): Laminar Flow Through a Porous Channel Bounded by Isothermal Parallel Plates, Int. J. Heat Mass Transfer 28, 851-858. doi:10.1016/0017-9310(85)90234-0

Krishna, D. V., Rao, P. N. and Sulochana, (2004): P. Hydro Magnetic Oscillatory Flow of a Second Order RivlinErickson Fluid in a Channel, Bull. Pure Appl. Sci. E 23(2), 291-303.

Jain, N. C., Gupta, P. and Sharma, B. (2006): Three Dimensional Couette Flow With Transpiration Cooling Through Porous Medium in Slip Flow Regime, AMSE J. Mod. Meas. Cont. B 75 (5), 33-52.

Sharma, P. R., Gaur, Y. N. and Sharma, R. P. (2005): Steady Laminar Flow And Heat Transfer of a Non-Newtonian Fluid Through a Straight Horizontal Porous Channel in The Presence of Heat Source, Ind. J. Theo. Phys. 53(1), 3747.

Sharma, P. R. and Yadav, G. R. (2005): Heat Transfer Through Three Dimensional Couette Flow Between a Stationary Porous Plate Bounded by Porous Medium and a Moving Porous Plate, Ultra Sci. Phys. Sci. 17(3M), 351360 .

Vajravelu, K. and Hadjinicolaou, A. (1993): Heat Transfer in a Viscous Fluid over a Stretching Sheet With Viscous Dissipation and Internal Heat Generation, Int. Commun. Heat Mass Transfer 20, 417-430. doi:10.1016/0735-1933(93)90026-R 